\begin{tabular}{|c|c|c|c|}
\hline 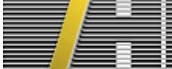 & 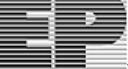 & PUBLISHED & 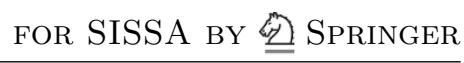 \\
\hline & & & $\begin{array}{l}\text { RECEIVED: December 18, } 2014 \\
\text { ACCEPTED: January 17, } 2015 \\
\text { Published: February 2, } 2015\end{array}$ \\
\hline
\end{tabular}

\title{
A cluster bootstrap for two-loop MHV amplitudes
}

\author{
John Golden and Marcus Spradlin \\ Department of Physics, Brown University, \\ Box 1843, Providence, RI 02912-1843, U.S.A. \\ E-mail: john_golden@brown.edu, marcus_spradlin@brown.edu
}

ABSTRACT: We apply a bootstrap procedure to two-loop MHV amplitudes in planar $\mathcal{N}=4$ super-Yang-Mills theory. We argue that the mathematically most complicated part (the $\Lambda^{2} B_{2}$ coproduct component) of the $n$-particle amplitude is uniquely determined by a simple cluster algebra property together with a few physical constraints (dihedral symmetry, analytic structure, supersymmetry, and well-defined collinear limits). We present a concise, closed-form expression which manifests these properties for all $n$.

KEYwords: Supersymmetric gauge theory, Scattering Amplitudes

ARXIV EPRINT: 1411.3289 


\section{Contents}

1 Cluster coordinates and coproducts 2

2 Elements of the cluster bootstrap $\quad 3$

$\begin{array}{lll}3 & \text { Applying the bootstrap } & 6\end{array}$

4 Discussion $\quad 7$

The scattering amplitudes of planar $\mathcal{N}=4$ supersymmetric Yang-Mills (SYM) theory [1] comprise a collection of functions with remarkable mathematical properties, tightly restricted by the physical constraints they must satisfy. Indeed the mathematical and physical properties of these amplitudes are, collectively, so restrictive that one marvels that the functions can even exist at all. The program of using known (or supposed) general properties of amplitudes to assemble concrete new results, which can then be verified by applying consistency checks, is generally known as the amplitude bootstrap. One of the ultimate goals of this program is to formulate a concise list of simple physical and mathematical constraints which might uniquely determine the precise functional form of all amplitudes in SYM theory.

The simplest incarnation of the bootstrap program applies to those $L$-loop $n$-particle $\mathrm{N}^{k} \mathrm{MHV}$ amplitudes which belong to the class of generalized polylogarithm functions [2]. All amplitudes with $L<2$ or $n<10$ or $k<3$ are believed [3] to belong to this class. Tools for dealing with the classes of functions which might appear in more general amplitudes are currently lacking, but there is every reason to suspect that a bootstrap program will forge ahead once the appropriate techniques are developed.

The essential tool for dealing with amplitudes of the generalized polylogarithm type is the symbol map (see [4]). The symbol of an amplitude of weight $w(=2 L)$ is an element of the $w$-fold tensor product of the multiplicative group generated by certain rational functions on the kinematic configuration space [5] $\operatorname{Conf}_{n}\left(\mathbb{P}^{3}\right)=\operatorname{Gr}(4, n) /\left(\mathbb{C}^{*}\right)^{n-1}$ for $n$ particle scattering in SYM theory. A fundamental working hypothesis of the bootstrap is that the set of rational functions allowed to appear in the symbol of any amplitude (i.e., the "symbol alphabet") is the set of $\mathcal{A}$-coordinates on the $\operatorname{Gr}(4, n)$ cluster algebra. Starting with the special collection of functions (or symbols) of this type, one bootstraps an amplitude by applying constraints and comparing with independent data from the literature (for example, from the amplitude OPE expansion [6-9], or from multi-Regge limits [1013]), until one arrives at a unique putative result. The cluster $\mathcal{A}$-coordinate hypothesis is supported by all explicit results for amplitudes available in the literature to date, including 
two-loop MHV for all $n$ [14], two-loop NMHV for $n=6,7[15,16]$, three-loop MHV and NMHV for $n=6[17,18]$, and four-loop MHV for $n=6$ [19].

Some recent investigations [5, 20-22] have revealed that the connection between the cluster structure [23] on $\operatorname{Conf}_{n}\left(\mathbb{P}^{3}\right)$ and the mathematical structure of amplitudes in SYM theory runs much deeper than merely specifying the appropriate symbol alphabet. We use the term "cluster bootstrap" to emphasize that our focus will be on understanding the implications of these "more clustery" properties, and in particular on how to harness their power via bootstrap. In this paper our attention is focused specifically on the planar $n$-particle two-loop MHV amplitudes $R_{n}^{(2)}$. We argue that these amplitudes are completely determined (modulo classical polylogarithm functions $\mathrm{Li}_{k}$ ) by a straightforward cluster property together with a few simple physical constraints. Consideration of these constraints leads us to the concise explicit formula (3.4) which specifies $R_{n}^{(2)}$ modulo $\mathrm{Li}_{k}$ 's.

\section{Cluster coordinates and coproducts}

Let us begin by recalling a few relevant facts about the $\operatorname{Gr}(4, n)$ Grassmannian cluster algebra. Physicists seeking additional background may find [5] useful. Cluster $\mathcal{X}$-coordinates are a preferred set of cross-ratios (dual conformally invariant [24] ratios of products of homogeneous polynomials in the $\mathcal{A}$-coordinates) on the kinematic domain $\operatorname{Conf}_{n}\left(\mathbb{P}^{3}\right)$. A "cluster" is a collection $\left\{x_{i}\right\}$ of $3(n-5)$ such coordinates with the property that the Poisson bracket matrix $B_{i j}=\left\{\log x_{i}, \log x_{j}\right\}$ has integer entries and maximal (if $n$ is odd) or nearly maximal (if $n$ is even) rank. Cluster $\mathcal{X}$-coordinates may be systematically constructed via a process called mutation, and a given $\mathcal{X}$-coordinate may appear in one or more clusters. For $n=6,7$ iterated application of mutations close on a finite set of clusters (14 and 833, respectively) containing a finite number of distinct $\mathcal{X}$-coordinates $(15,385)$.

For $n>7$ one can mutate indefinitely to produce an infinite number of $\mathcal{A}$ - and $\mathcal{X}$ coordinates, but this poses no conceptual obstacle to the bootstrap program since only finitely many can appear in any individual generalized polylogarithm function (i.e., at any finite loop order). For example, the symbol of $R_{n}^{(2)}$ contains $\frac{n}{2}\left(3 n^{2}-30 n+77\right) \mathcal{A}$-coordinates. These can easily be enumerated by inspecting the all- $n$ result of [14]: in the notation of that paper, there are $n(n-6)$ symbol letters of the form $\langle 1(23)(n-1 n)(i i+1)\rangle$ (plus all cyclic partners), $\frac{n}{2}(n-6)(n-7)$ of the form $\langle 12 \bar{i} \cap \bar{j}\rangle$ (plus cyclic), and $\frac{n}{2}(n-5)(n-6)$ of the form $\langle 1(n 2)(i i+1)(j j+1)\rangle$ (plus cyclic). Finally, there are of course the simple Plücker coordinates $\langle i j k l\rangle$, which number $\left(\begin{array}{l}n \\ 4\end{array}\right)$; however it is evident from [14] that the only ones which appear in the two-loop MHV amplitudes are those in which at least one pair among $i j, j k, k l$ or $l i$ are cyclically adjacent (for example, $\langle 1357\rangle$ does not appear for $n>7$ ), so we must subtract $\frac{n}{24}(n-5)(n-6)(n-7)$ from $\left(\begin{array}{l}n \\ 4\end{array}\right)$. Adding up all of these types we find a total of $\frac{n}{2}\left(3 n^{2}-30 n+77\right)$ symbol letters.

To determine whether a dual conformal cross-ratio $R$ formed from these letters is an $\mathcal{X}$ coordinate, we apply a simple heuristic, originally described in [5, 21]: $R$ is an $\mathcal{X}$-coordinate if $1+R$ can also be expressed as a ratio of products of letters and if $R$ is positive everywhere inside the positive domain (i.e., the domain in which $\langle i j k l\rangle>0$ for all $i<j<k<l$ ). We know of no example where this heuristic fails. 
Although the symbol of $R_{n}^{(2)}$ is known for all $n$ [14], explicit analytic results for the function $R_{n}^{(2)}$ are available only for $n=6,7[22,25]$. While obtaining more general fully analytic results is certainly a worthwhile goal, in order to cut to the core of the mathematical structure of these functions it is natural to focus on the coproduct (or, more properly, the cobracket [5])

$$
\delta\left(R_{n}^{(2)}\right) \in \Lambda^{2} \mathrm{~B}_{2} \bigoplus \mathrm{B}_{3} \otimes \mathbb{C}^{*}
$$

We remind the reader that the Bloch group $\mathrm{B}_{n}$ is generated by elements denoted $\{x\}_{n}$. Concretely, $\{x\}_{n}$ denotes the equivalence class of weight- $n$ functions, modulo products, containing $-\mathrm{Li}_{n}(-x)$. Any generalized polylogarithm of weight 4 is determined, modulo products of functions of lower weight, by the two coproduct components shown above. Moreover the $\Lambda^{2} \mathrm{~B}_{2}$ component captures the "most nontrivial" part of a function and determines the $\mathrm{B}_{3} \otimes \mathbb{C}^{*}$ component modulo terms involving the quadrilogarithm function $\mathrm{Li}_{4}[26,27]$.

In principle one could compute the coproduct $\delta\left(R_{n}^{(2)}\right)$ directly from the known symbol of $R_{n}^{(2)}$. However, the representation of the symbol given in [14] does not have the appropriate cluster structure manifest, making such a calculation infeasible. Instead we put aside our knowledge of the symbol for a moment while we bootstrap our way towards an explicit formula for $\left.\delta\left(R_{n}^{(2)}\right)\right|_{\Lambda^{2} \mathrm{~B}_{2}}$, shown in eq. (3.4). While this formula is strictly speaking conjectural, being based on some presumed cluster algebra structure of the amplitude, we have checked it by explicit comparison to the results of [14] through $n=13$.

\section{Elements of the cluster bootstrap}

The structure of MHV scattering amplitudes is heavily constrained at the level of the symbol, a fact which Dixon and collaborators have exploited to great effect (see [28] for a recent review on the $n=6$ bootstrap). We wish to adopt a similar approach at the level of the coproduct, specifically the $\Lambda^{2} \mathrm{~B}_{2}$ component. To this end we start by formulating a hypothesis for what kinds of variables $x, y$ the cluster bootstrap should allow to appear in $\{x\}_{2} \wedge\{y\}_{2}$. It has been observed "experimentally", for small values of $n$ [5], that the twoloop MHV amplitudes have the very special feature that their $\Lambda^{2} \mathrm{~B}_{2}$ coproduct components are always expressible in terms of linear combinations of terms $\{v\}_{2} \wedge\{z\}_{2}$ where

1. each $v$ is drawn from a set of cluster $\mathcal{X}$-coordinates specially adapted to the analytic structure of the amplitude (related to what is called the "first-entry condition"),

2. each $z$ is drawn from a set of cluster $\mathcal{X}$-coordinates specially adapted to the supersymmetry properties of MHV amplitudes (related to what is called the "last-entry condition"),

3. and the two variables $v, z$ appearing in each term always belong to at least one cluster in common (this means, in particular, that $\{\log v, \log z\} \in\{-1,0,+1\}$ with respect to the natural Poisson bracket on the kinematic manifold $\left.\operatorname{Conf}_{n}\left(\mathbb{P}^{3}\right)\right)$.

Let us explain these points in a little more detail. 
First we recall that the analytic structure of color-ordered scattering amplitudes is highly constrained: they may only have branch points on the boundary of the Euclidean region at points where some sum of cyclically adjacent momenta becomes null. Requiring that amplitudes have only physical singularities implies that the first entries of the symbol of any amplitude must be drawn from the set of cross-ratios given by

$$
u_{i j}=\frac{\langle i i+1 j+1 j+2\rangle\langle i+1 i+2 j j+1\rangle}{\langle i i+1 j j+1\rangle\langle i+1 i+2 j+1 j+2\rangle} .
$$

Unfortunately, none of the $u_{i j}$ are cluster $\mathcal{X}$-coordinates. Instead we consider the closely related quantities

$$
v_{i j k}=\frac{1}{\prod_{a=j}^{k-1} u_{i a}}-1=-\frac{\langle i+1(i i+2)(j j+1)(k k+1)\rangle}{\langle i i+1 k k+1\rangle\langle i+1 i+2 j j+1\rangle},
$$

where

$$
\langle a(b c)(d e)(f g)\rangle \equiv\langle a b d e\rangle\langle a c f g\rangle-\langle a b f g\rangle\langle a c d e\rangle .
$$

$v_{i j k}$ is a $\mathcal{X}$-coordinates as long as $i<j<k(\bmod n)$. We can phrase the familiar first-entry condition in terms of these unfamiliar variables by saying that only the quantities $1+v_{i j k}$ are allowed in the first entry of the symbol of any function with physical branch cuts.

Secondly we recall the MHV last-entry condition [16], which states that the last entry of the symbol of any MHV amplitude must, as a consequence of extended supersymmetry, be drawn from the set of Pluckker coordinates of the form $\langle\bar{i} j\rangle \equiv\langle i-1 i i+1 j\rangle$. We therefore might like to include ratios built purely out of these objects in our ansatz. Unfortunately, no $\mathcal{X}$-coordinates of this type exist. Instead we consider the cross-ratios

$$
z_{i j k}^{+}=\frac{\langle i i+1 \bar{j} \cap \bar{k}\rangle}{\langle i \bar{k}\rangle\langle i+1 \bar{j}\rangle}, \quad z_{i j k}^{-}=\frac{\langle i i+1 j k\rangle\langle\bar{i} i+2\rangle}{\langle\bar{i} k\rangle\langle\overline{i+1} j\rangle},
$$

where

$$
\langle a b \bar{c} \cap \bar{d}\rangle \equiv\langle a \bar{c}\rangle\langle b \bar{d}\rangle-\langle b \bar{c}\rangle\langle a \bar{d}\rangle .
$$

The $z_{i j k}^{ \pm}$are all cluster $\mathcal{X}$-coordinates for $\operatorname{Gr}(4, n)$ as long as $i<j<k(\bmod n)$, and as suggested by the notation, $z_{i j k}^{ \pm}$are parity conjugates of each other (see [5] for a discussion of parity on $\operatorname{Conf}_{n}\left(\mathbb{P}^{3}\right)$ ). Despite appearances these are in fact intimately tied to the last-entry condition since

$$
1+z_{i j k}^{+}=\frac{\langle i \bar{j}\rangle\langle i+1 \bar{k}\rangle}{\langle i \bar{k}\rangle\langle i+1 \bar{j}\rangle}, \quad 1+z_{i j k}^{-}=\frac{\langle\bar{i} j\rangle\langle\overline{i+1} k\rangle}{\langle\bar{i} k\rangle\langle\overline{i+1} j\rangle} .
$$

It is useful to define certain boundary cases of the above cross-ratios with overlapping indices:

$$
v_{i j}=v_{i j j+1}, \quad z_{i j}=z_{i j j+1}^{-},
$$

where parity takes $z_{i j} \rightarrow z_{j i}$. Similar to what was done in the previous paragraph, we may express the familiar last-entry condition in terms of these unfamiliar variables by saying that only the quantities $1+z_{i j k}^{ \pm}$are allowed in the final entry of the symbol of any MHV amplitude. 
Third, it is worth commenting on how the Poisson bracket $\{\log x, \log y\}$ between two $\mathcal{X}$-coordinates may be computed in practice. If one could enumerate all possible clusters (and the Poisson bracket matrix in each cluster), by repeated application of the mutation algorithm starting with the initial cluster reviewed in [22], then one could scan that list to determine whether or not a given pair $x, y$ appears together inside any cluster (and, if so, then one could read off their Poisson bracket). For the infinite algebras we encounter when $n>7$ it is obviously not feasible to enumerate all clusters. An alternative approach would be to express $x$ and $y$ as algebraic functions of the $\mathcal{X}$-coordinates $\left(u_{1}, u_{2}, \ldots\right)$ in the initial cluster and then to compute

$$
\{\log x, \log y\}=\sum_{i, j} \frac{\partial \log x}{\partial \log u_{i}} \frac{\partial \log y}{\partial \log u_{j}}\left\{\log u_{i}, \log u_{j}\right\} .
$$

If for a given pair $x, y$ the right-hand side comes out to be 0 or \pm 1 , then it is guaranteed that there exists a cluster containing both $x$ and $y$, even if it would be computationally infeasible to find a specific path of mutations connecting that cluster to the initial cluster. However, we have found that the simplest way to compute $\{\log x, \log y\}$ for general $x, y$ is to use the fact that the Poisson bracket on $\operatorname{Gr}(k, n)$ is induced from the easily computible Sklyanin bracket on $\mathrm{SL}_{n}$, as described for example in [29]. ${ }^{1}$

The collection of all $\frac{1}{2} n(n-5)^{2}$ of the $v$ 's and $n(n-5)^{2}$ of the $z$ 's constitutes what we call the $\{v, z\}$ basis. Noting that $\{1+x\}_{2}=-\{x\}_{2}$, the discussion in the previous two paragraphs suggests that it is natural to seek a representation for $\left.\delta\left(R_{n}^{(2)}\right)\right|_{\Lambda^{2} \mathrm{~B}_{2}}$ as a linear combination of objects of the form $\{v\}_{2} \wedge\{z\}_{2}$ which capture, at the level of the coproduct, the spirit of both the first- and last-entry constraints satisfied by the symbol. Of course, the $\wedge$-product obscures any precise notion of first or last entries for the coproduct, so our argument for restricting to $\{v\}_{2} \wedge\{z\}_{2}$ is meant to be suggestive rather than rigorous. The suitability of this ansatz is justified a posteriori because it leads to a successful bootstrap.

Based on these considerations, as well as explicit calculations at small $n$, we are motivated to hypothesize that properties $1-3$ listed above are true for general $n$, so we adopt these as core elements of the cluster bootstrap for $R_{n}^{(2)}$. In addition we impose that $R_{n}^{(2)}$ should be

4. invariant under the dihedral group acting on the $n$ particle labels, as well as under parity, and

5. well-defined under collinear limits.

We have found that these five simple physical and mathematical conditions uniquely fix $\left.\delta\left(R_{n}^{(2)}\right)\right|_{\Lambda^{2} \mathrm{~B}_{2}}$ (up to a single overall multiplicative constant common to all $n$ ) to take the value shown explicitly in eq. (3.4). Let us emphasize that in step 5 it is not actually necessary to know the $n$-1-particle result in order to construct the answer for $n$ particles; it is sufficient merely to make an appropriate ansatz for the latter and impose only that the $n \| n-1$ collinear limit is well-defined. This determines both the $n$ - and $n-1$-particle results at the same time, and in particular ties together their overall normalizations.

\footnotetext{
${ }^{1}$ We are very grateful to C. Vergu for pointing out this method to us.
} 


\section{Applying the bootstrap}

Let us explain in some detail how the procedure works beginning with $n=7$. In this case there are are $14 v$ 's and $28 z$ 's, so we start with the ansatz that $\left.\delta\left(R_{7}^{(2)}\right)\right|_{\Lambda^{2} \mathrm{~B}_{2}}$ should be a linear combination of the $14 \times 28=392$ possible $\{v\}_{2} \wedge\{z\}_{2}$ 's. Only 70 of these pairs have Poisson brackets in the set $\{-1,0,+1\}$ (i.e., appear together in a cluster), and after imposing dihedral and parity symmetries we are left with the three-parameter ansatz

$$
\left(c_{1}\left\{v_{14}\right\}_{2} \wedge\left\{z_{14}\right\}_{2}+c_{2}\left\{v_{14}\right\}_{2} \wedge\left\{z_{15}\right\}_{2}+c_{3}\left\{v_{146}\right\}_{2} \wedge\left\{z_{624}^{-}\right\}_{2}\right)+\text { dihedral + conjugate }
$$

We then take the collinear limit parameterized by $Z_{n} \rightarrow Z_{n-1}+\alpha\left(Z_{n-2}+\beta Z_{1}\right)+\gamma Z_{2}$ with $\gamma \rightarrow 0$, then $\alpha \rightarrow 0$, leaving $\beta$ free. This leads to

$$
\begin{aligned}
& c_{2}\left(\left(\left\{v_{25} v_{2 \beta} /\left(1+v_{2 \beta}\right)\right\}_{2}+\left\{v_{25} /\left(1+v_{3 \beta}\right)\right\}_{2}\right) \wedge\left\{z_{36}\right\}_{2}\right. \\
&\left.\quad+\left\{v_{14}\right\}_{2} \wedge\left(\left\{z_{14}\right\}_{2}+\left\{z_{25}\right\}_{2}\right)+\left\{v_{36}\right\}_{2} \wedge\left(\left\{z_{14}\right\}_{2}+\left\{z_{36}\right\}_{2}\right)\right) \\
& \quad+\left(c_{1}-c_{3}\right)\left(\left\{v_{2 \beta}\right\}_{2} \wedge\left\{z_{25}\right\}_{2}-\left\{v_{3 \beta}\right\}_{2} \wedge\left\{z_{36}\right\}_{2}\right)+\text { conjugate },
\end{aligned}
$$

where $v_{i \beta}=\beta\langle 1 i i+16\rangle /\langle i i+156\rangle$ and "+ conjugate" applies to the entire expression. For the collinear limit to be well-defined, i.e., independent of $\beta$ (which specifies the relative length of the collinear momenta 6 and 7 ), we require $c_{1}=c_{3}$ and $c_{2}=0$. We have therefore determined that $\left.\delta\left(R_{6}^{(2)}\right)\right|_{\Lambda^{2} \mathrm{~B}_{2}}=0$ and also reproduce the result [5] that

$$
\left.\delta\left(R_{7}^{(2)}\right)\right|_{\Lambda^{2} \mathrm{~B}_{2}}=\left\{v_{14}\right\}_{2} \wedge\left\{z_{14}\right\}_{2}+\left\{v_{146}\right\}_{2} \wedge\left\{z_{624}^{-}\right\}_{2}+\text { dihedral }+ \text { conjugate },
$$

up to an overall multiplicative constant.

The analogous ansatz for $n=8$ begins with $36 \times 72=2592$ terms of the form $\{v\}_{2} \wedge\{z\}_{2}$. Restricting to pairs that appear together in a cluster reduces this to 400. After imposing the discrete symmetries only 15 free parameters remain, and requiring the $8 \| 7$ collinear limit to be well-defined fixes all of them up to an overall normalization, which in turn may be fixed by matching eq. (3.3). The bootstrap may be carried out through sufficiently large $n$ to motivate the all- $n$ conjecture

$$
\begin{aligned}
\left.\delta\left(R_{n}^{(2)}\right)\right|_{\Lambda^{2} \mathrm{~B}_{2}}= & \sum_{1<i<j<n}\left(\left\{v_{1 i j}\right\}_{2} \wedge\left(-\left\{z_{i j}\right\}_{2}-\left\{z_{j 2 i}^{-}\right\}_{2}+\left\{z_{i j 2}^{-}\right\}_{2}+\left\{z_{j 2 i+1}^{-}\right\}_{2}-\left\{z_{i j+12}^{-}\right\}_{2}\right)\right. \\
& \left.-\left\{v_{1 i}\right\}_{2} \wedge\left(\left\{z_{j 2 i+1}^{-}\right\}_{2}+\sum_{j<k \leq 1}\left\{z_{j k}\right\}_{2}\right)+\text { cyclic }+ \text { conjugate }\right) .
\end{aligned}
$$

Here "+ cyclic + conjugate" applies to the both lines, and we note that eq. (3.4) does satisfy the full dihedral symmetry even though we have only chosen to manifest + cyclic. The multiple sums contain some boundary terms which evaluate to $\{0\}_{2}$ or $\{\infty\}_{2}$; these are understood to be omitted. We have explicitly checked (by comparing its iterated coproduct) that this expression is consistent with the known symbol of $R_{n}^{(2)}$ through $n=13$. 


\section{Discussion}

A striking and mysterious feature of eq. (3.4) is that all of the pairs $\{v, z\}$ appearing in the formula have Poisson bracket zero. This feature is an output of the bootstrap; the input was much weaker, with the initial ansatz also allowing pairs having Poisson bracket $\{\log v, \log z\}= \pm 1$. Geometrically, this means that the $\Lambda^{2} \mathrm{~B}_{2}$ coproduct component wants to be expressed in terms of quadrilateral (rather than pentagonal) dimension- 2 faces of the generalized Stasheff polytope associated to the $\operatorname{Gr}(4, n)$ cluster algebra, as noted already in [5].

It would naturally be interesting to formulate a bootstrap for computing the $\mathrm{B}_{3} \otimes \mathbb{C}^{*}$ coproduct components of $R_{n}^{(2)}$, which contain information about $\mathrm{Li}_{4}$ terms that $\Lambda^{2} \mathrm{~B}_{2}$ does not know about. Unfortunately we have found that the $\{v, z\}$ basis provides an insufficient ansatz for $\mathrm{B}_{3} \otimes \mathbb{C}^{*}$ already at $n=7$. Of course there is no obstacle to computing this coproduct component on a case by case basis for small $n$ by starting with a larger collection of cluster $\mathcal{X}$-coordinates, but deriving (or even guessing) an all- $n$ formula remains elusive.

It would also be interesting to extend eq. (3.4) to capture more or even all of $R_{n}^{(2)}$, including terms involving products of $\mathrm{Li}_{k}$ 's. Interestingly we have found, using the standard symbol-level (anti-)symmetrization techniques outlined in [25], that all of the $\mathrm{Li}_{2} \mathrm{Li}_{2}$ terms in $R_{n}^{(2)}$ are captured by the remarkable formula

$$
\text { "eq. (3.4)" - }\left(\sum_{1 \leq i<j \leq n} \operatorname{Li}_{2}\left(-v_{i j}\right)\right)^{2},
$$

where the first term refers to eq. (3.4) with the replacement $\{a\}_{2} \wedge\{b\}_{2} \rightarrow \operatorname{Li}_{2}(-a) \operatorname{Li}_{2}(-b)$. This is a strong indication that there is still more structure to discover in the two-loop $n$ particle MHV amplitudes.

Surely the most important and interesting open question is whether a suitable "cluster bootstrap" can be formulated for higher-loop MHV or non-MHV amplitudes. The main obstacle is that so few explicit results for such amplitudes are known, even just at the level of symbols, that we do not yet dare to speculate how elements 1-3 of the cluster bootstrap ought to be generalized.

\section{Acknowledgments}

We have benefitted from stimulating discussions and collaboration on closely related topics with J. Drummond, G. Papathanasiou, M. Paulos, C. Vergu, A. Volovich, and especially A. Goncharov. This work was supported by the US Department of Energy under contract DE-SC0010010.

Open Access. This article is distributed under the terms of the Creative Commons Attribution License (CC-BY 4.0), which permits any use, distribution and reproduction in any medium, provided the original author(s) and source are credited. 


\section{References}

[1] L. Brink, J.H. Schwarz and J. Scherk, Supersymmetric Yang-Mills Theories, Nucl.Phys. B 121 (1977) 77 [INSPIRE].

[2] A.B. Goncharov, Galois symmetries of fundamental groupoids and noncommutative geometry, Duke Math. J. 128 (2005) 209 [math/0208144].

[3] N. Arkani-Hamed, J.L. Bourjaily, F. Cachazo, A.B. Goncharov, A. Postnikov et al., Scattering Amplitudes and the Positive Grassmannian, arXiv: 1212.5605 [INSPIRE].

[4] A.B. Goncharov, A simple construction of Grassmannian polylogarithms, arXiv:0908.2238 [INSPIRE].

[5] J. Golden, A.B. Goncharov, M. Spradlin, C. Vergu and A. Volovich, Motivic Amplitudes and Cluster Coordinates, JHEP 01 (2014) 091 [arXiv: 1305.1617] [INSPIRE].

[6] B. Basso, A. Sever and P. Vieira, Spacetime and Flux Tube S-Matrices at Finite Coupling for $N=4$ Supersymmetric Yang-Mills Theory, Phys. Rev. Lett. 111 (2013) 091602 [arXiv: 1303.1396] [INSPIRE].

[7] A. Sever, P. Vieira and T. Wang, OPE for Super Loops, JHEP 11 (2011) 051 [arXiv:1108.1575] [INSPIRE].

[8] D. Gaiotto, J. Maldacena, A. Sever and P. Vieira, Pulling the straps of polygons, JHEP 12 (2011) 011 [arXiv:1102.0062] [InSPIRE].

[9] L.F. Alday, D. Gaiotto, J. Maldacena, A. Sever and P. Vieira, An Operator Product Expansion for Polygonal null Wilson Loops, JHEP 04 (2011) 088 [arXiv:1006.2788] [INSPIRE].

[10] J. Bartels, L.N. Lipatov and A. Sabio Vera, BFKL Pomeron, Reggeized gluons and Bern-Dixon-Smirnov amplitudes, Phys. Rev. D 80 (2009) 045002 [arXiv:0802.2065] [INSPIRE].

[11] J. Bartels, L.N. Lipatov and A. Sabio Vera, N=4 supersymmetric Yang-Mills scattering amplitudes at high energies: The Regge cut contribution, Eur. Phys. J. C 65 (2010) 587 [arXiv:0807.0894] [INSPIRE].

[12] V.S. Fadin and L.N. Lipatov, BFKL equation for the adjoint representation of the gauge group in the next-to-leading approximation at $N=4$ SUSY, Phys. Lett. B 706 (2012) 470 [arXiv: 1111.0782] [INSPIRE].

[13] L.N. Lipatov, A. Prygarin and H.J. Schnitzer, The Multi-Regge limit of NMHV Amplitudes in $N=4$ SYM Theory, JHEP 01 (2013) 068 [arXiv:1205.0186] [INSPIRE].

[14] S. Caron-Huot, Superconformal symmetry and two-loop amplitudes in planar $N=4$ super Yang-Mills, JHEP 12 (2011) 066 [arXiv:1105.5606] [INSPIRE].

[15] L.J. Dixon, J.M. Drummond and J.M. Henn, Analytic result for the two-loop six-point NMHV amplitude in $N=4$ super Yang-Mills theory, JHEP 01 (2012) 024 [arXiv:1111.1704] [INSPIRE].

[16] S. Caron-Huot and S. He, Jumpstarting the All-Loop S-matrix of Planar $N=4$ Super Yang-Mills, JHEP 07 (2012) 174 [arXiv:1112.1060] [INSPIRE].

[17] L.J. Dixon and M. von Hippel, Bootstrapping an NMHV amplitude through three loops, JHEP 10 (2014) 065 [arXiv:1408.1505] [INSPIRE]. 
[18] L.J. Dixon, J.M. Drummond, M. von Hippel and J. Pennington, Hexagon functions and the three-loop remainder function, JHEP 12 (2013) 049 [arXiv:1308.2276] [INSPIRE].

[19] L.J. Dixon, J.M. Drummond, C. Duhr and J. Pennington, The four-loop remainder function and multi-Regge behavior at NNLLA in planar $N=4$ super-Yang-Mills theory, JHEP 06 (2014) 116 [arXiv: 1402.3300] [INSPIRE].

[20] J. Golden, M.F. Paulos, M. Spradlin and A. Volovich, Cluster Polylogarithms for Scattering Amplitudes, J. Phys. A 47 (2014) 474005 [arXiv: 1401.6446] [InSPIRE].

[21] J. Golden and M. Spradlin, The differential of all two-loop $M H V$ amplitudes in $\mathcal{N}=4$ Yang-Mills theory, JHEP 09 (2013) 111 [arXiv:1306.1833] [INSPIRE].

[22] J. Golden and M. Spradlin, An analytic result for the two-loop seven-point MHV amplitude in $\mathcal{N}=4$ SYM, JHEP 08 (2014) 154 [arXiv: 1406.2055] [INSPIRE].

[23] V.V. Fock and A.B. Goncharov, Cluster ensembles, quantization and the dilogarithm, Ann. Sci. Éc. Norm. Supér. 42 (2009) 865 [math/0311245] [INSPIRE].

[24] J.M. Drummond, J. Henn, G.P. Korchemsky and E. Sokatchev, Dual superconformal symmetry of scattering amplitudes in $N=4$ super-Yang-Mills theory, Nucl. Phys. B 828 (2010) 317 [arXiv:0807.1095] [INSPIRE].

[25] A.B. Goncharov, M. Spradlin, C. Vergu and A. Volovich, Classical Polylogarithms for Amplitudes and Wilson Loops, Phys. Rev. Lett. 105 (2010) 151605 [arXiv:1006.5703] [INSPIRE].

[26] A.B. Goncharov, Polylogarithms and motivic Galois groups, in Motives (Seattle WA, 1991), Proc. Sympos. Pure Math. 55, pg. 43-96, [Amer. Math. Soc., Providence RI U.S.A. (1994)].

[27] A.B. Goncharov, Geometry of Configurations, Polylogarithms, and Motivic Cohomology, Adv. Math. 114 (1995) 197.

[28] L.J. Dixon, J.M. Drummond, C. Duhr, M. von Hippel and J. Pennington, Bootstrapping six-gluon scattering in planar $N=4$ super-Yang-Mills theory, PoS(LL2014) 077 [arXiv: 1407 . 4724] [INSPIRE].

[29] M. Gekhtman, M. Shapiro and A. Vainshtein, Cluster algebras and Poisson geometry, Mosc. Math. J. 3 (2003) 899. 